\title{
La controversia de la inmigración internacional: desafíos para el Trabajo Social en EE.UU. y Chile
}

\section{The controversy of International Immigration: Challenges to Social Work in the U.S.A and Chile}

Darío Menanteau

Profesor de Sociología, Trabajo Social y Derechos Humanos University of Minnesota. Dirección postal 225 Peters Hall 1404 Gortner Avenue; St.Paul, Minnesota 55108 - USA.E-mail:dmenante@umn.edu

\begin{abstract}
Resumen
La inmigración internacional es uno de los procesos socio-demográficos de mayor trascendencia e impacto en el mundo contemporáneo. Sin embargo, a pesar de su importancia, ni los modelos teóricos utilizados en su estudio, ni las políticas públicas o leyes reguladoras logran explicar o eliminar estereotipos de rechazo y exclusión hacia los inmigrantes.

Este artículo analiza la presencia de algunas actitudes y conductas negativas observadas en los Estados Unidos hacia la población hispana y algunas expresiones similares en Chile, en relación a inmigrantes provenientes del Perú. El estudio sugiere que este es un fenómeno universal el cual requiere una perspectiva teórica amplia que involucre el sistema social en su totalidad. El desafío que esto significa, tanto para las ciencias sociales como para los profesionales en el campo del trabajo social, implica investigaciones y actividades que permitan incorporar la migración a las estrategias y tareas del desarrollo nacional.
\end{abstract}

Palabras claves. Migración, inmigración, estereotipos, desarrollo social.

\begin{abstract}
International immigration is one of the socio-demographic processes of great consequence and impact in the world today. Despite its importance, however, neither theoretical models, public policies, nor legislation are able to explain or eliminate negative stereotypes or patterns of behavior expressing rejection and exclusion towards immigrants.

The article analyzes the presence of some negative attitudes and behavior observed in the United States towards Hispanics and some similar expressions in Chile regarding immigrants from Peru. The study suggests that this is a universal phenomenon which requires a theoretical perspective that includes the entire social systems. The challenge of this to the social sciences and to professional social workers implies further research and activities to better incorporate migration into the strategies and tasks of national development.
\end{abstract}

Key words. Migration, immigration, stereotypes, social development.

\section{Introducción}

Uno de los resultados más visibles de la globalización es el creciente movimiento demográfico entre países. Las fronteras que en el pasado separaban continentes y naciones, han disminuido en la medida que la economía internacional es más global e interdependiente. La nueva globalización, no sólo favorece el intercambio de capitales, productos y tecnologías entre países, sino que ha estimulado también el traslado de personas y la aparición de nuevas corrientes migratorias.
Las exigencias del comercio, la industria, transportes y comunicaciones no sólo han facilitado el intercambio de productos y la inversión de recursos financieros entre naciones sino que también, han promovido los contactos e intercambio de personas entre diferentes países.

Para quienes favorecen el proceso de globalización, señalan que las nuevas tecnologías y su rápida difusión por el planeta, podrían aportar también grandes beneficios (al menos potenciales) para todos los países. Según ellos, la economía internacional po- 
dría ayudar a generar una mayor productividad y mejor distribución de recursos para la alimentación, salud, educación y bienestar de los pueblos.

Para otros investigadores, sin embargo, la realidad económica mundial demuestra que la globalización no ha resultado ser compatible con las necesidades de desarrollo nacional en la mayoría de los países, sean estos del 'primer' o del 'tercer' mundo. Al fin y al cabo, ambos mundos tienen serias necesidades de mejorar las condiciones de vida de vastos sectores de su población. (Naciones Unidas, 1998).

En relación a las consecuencias que emanan de la globalización, el escritor Mexicano Carlos Fuentes (2000), indica que la globalización ofrece, por una parte, la capacidad de "crear prosperidad, pero también exclusión y la creación de una sub-clase permanente." Fuentes sostiene que la globalización puede exacerbar las desigualdades y duplicar los niveles de pobreza en el mundo durante las próximas décadas.

Este proceso de globalización que se caracteriza por la ampliación de los mercados internacionales, la concentración del poder financiero y la presencia de grandes corporaciones transnacionales, - lejos de beneficiar a todos equitativamente-, genera condiciones de vida insostenibles para los países más pobres y para grupos humanos que quedan marginados de los frutos y beneficios de la globalización. Para estos últimos, la alternativa es emigrar a otros países, con la esperanza de encontrar un trabajo que les permita satisfacer sus necesidades básicas y las de sus respectivas familias.

Esta situación es la que se observa actualmente en diferentes países del mundo en desarrollo, donde muchos de sus habitantes no disponen de los medios necesarios para subsistir decentemente y se ven forzados a emigrar. Ocurre, por ejemplo, en varios países Europeos que reciben gran número de inmigrantes, tanto legales como indocumentados, de países con menos recursos. También, en los Estados Unidos, la inmigración desde México, Centro América y el Caribe, ha crecido en las últimas décadas, como además ha ocurrido con el número de inmigrantes y refugiados de Vietnam y de algunos países Africanos como Etiopía y Somalia.

En América Latina se observa cierto crecimiento de migrantes hacia países donde un mayor grado de estabilidad política y crecimiento económico ofrecen mejores oportunidades de subsistencia y mejoramiento de vida que en sus propios países de origen. En la actualidad, este podría ser el caso de la inmigración peruana a Chile.
Este artículo analiza algunos antecedentes relacionados con la actual controversia que suscita la inmigración internacional en algunos países:

1. Primero, se describen brevemente algunas perspectivas conceptuales sobre la inmigración y los procesos de asimilación, aceptación y rechazo a los migrantes;

2. Luego, se analiza algunos aspectos de la presencia Hispana o Latina en los Estados Unidos y se discuten algunos resultados de recientes investigaciones que podrían ayudar a erradicar mitos y des-información sobre los problemas y desventajas del movimiento poblacional entre países de las Américas.

3. El artículo subraya la importancia de las ciencias sociales y las funciones de servicio y de investigación de profesionales en el campo del Trabajo Social para reunir información válida sobre los aportes y necesidades de los inmigrantes. Este tipo de información podría ayudar a modificar actitudes y conductas xenofóbicas en países receptores de inmigrantes, como es el caso de los Estados Unidos respecto a los hispanos y el caso de Chile con inmigrantes peruanos.

\section{Perspectivas conceptuales sobre inmigración}

Los movimientos migratorios han despertado gran interés entre científicos sociales preocupados de analizar los procesos e impactos de la migración en el sistema social internacional. Aparece relevante, sin embargo, que entre algunas de las primeras teorías que se abocaron a estos estudios dieron marcada prioridad a los procesos de adaptación de los inmigrantes a un nuevo medio socio-cultural. Para esto, pusieron énfasis en los mecanismos de socialización para desarrollar una integración exitosa del inmigrante. (Handlin, 1941; Portes and Manning, 1986).

\section{La perspectiva de la asimilación cultural}

Este marco teórico fue propuesto inicialmente por Robert Park (1926) en su obra Raza y Cultura. En ella sugiere que la adaptación depende fundamentalmente del aprendizaje por parte del inmigrante de valores, normas y conductas aceptables en la cultura receptora. Este autor se refiere a la importancia del contexto social y a situaciones locales que tienden a originar problemas de relación entre diversos grupos étnicos. Asimismo, Park sostiene que como la naturaleza de las relaciones étnicas son principalmente cambiantes, ellas tienden a mejorar con el 
tiempo a medida que los miembros de la minoría se adaptan a la nueva cultura.

Myrdal (1944) expande esta noción de la asimilación cultural de grupos minoritarios, argumentando que en la experiencia de relaciones étnicas se produce un cambio cultural que afecta, simultáneamente, tanto a los miembros de la sociedad dominante como a las poblaciones minoritarias. Según esta perspectiva, los inmigrantes se adaptan a los patrones culturales, valores, normas y conductas de la nueva sociedad (Gordon, 1964). En la medida que los inmigrantes se familiarizan con el nuevo sistema social y se adaptan a las expectativas y practicas locales de la nueva comunidad nacional, es posible (al menos en teoría) que ellos sean víctimas de menor discriminación logrando así aceptación y acceso a mejores oportunidades para una movilidad económica y social en el nuevo medio. Para Berry (1993:278), sin embargo, para tener buen éxito en este proceso, sería necesario que los inmigrantes renuncien voluntariamente a sus valores tradicionales y costumbres ancestrales. Sólo así, los nuevos inmigrantes podrían alcanzar una completa, o al menos más amplia aceptación por parte de los miembros de la cultura dominante.

\section{El paradigma multicultural}

Esta perspectiva ofrece una visión alternativa a las exigencias propuestas por la teoría de la asimilación cultural antes descrita. Quienes postulan el concepto del Paradigma Multicultural sostienen que la relación de diversos grupos étnicos o nacionales implica un cierto grado de intercambios mediante los cuales los individuos se las arreglan para mantener elementos de su propia identidad étnica. (Berry 1993:277-78). Este proceso es lo que permitiría desarrollar un mayor grado de pluralismo sociocultural en una sociedad cada vez más pluralista y diversa. (Glazer y Moynihan 1970; Alba and Chamlin 1983)

\section{El concepto del sistema económico mundial}

Esta perspectiva analiza los movimientos migratorios desde el punto de vista del desplazamiento laboral en algunos países y la demanda de trabajadores en otros. La amplitud y énfasis de este modelo abarca todo el sistema de la economía internacional. De acuerdo a este modelo, la incorporación de los inmigrantes a un nuevo mercado laboral es una respuesta fundamental a los cambios y desplazamientos geográficos del capital transnacional. (Castells 1975, Portes 1978, Sassen-Koob (1978). De esta manera, en un mercado laboral segmentado, las necesidades de rebajar los costos y aumentar las ganancias se traduce en una mayor demanda de trabajadores con bajos salarios, se recurre a dar ocupación a los trabajadores migrantes y estacionales. La concentración de inmigrantes en este tipo de ocupaciones que usualmente requieren bajos niveles de educación y son pobremente remunerados, se traduce en condiciones de oportunidades limitadas $y$ altos niveles de pobreza entre estos inmigrantes. De acuerdo a esto, se podría argumentar que este mecanismo operacional de la economía capitalista mundial mantiene las minorías étnicas en los tipos de trabajo extractivos y manufactureros lo que es altamente funcional y conveniente para las necesidades de los grupos dominantes.

\section{La red de relaciones étnicas}

Según esta perspectiva, los inmigrantes junto a sus valores y prácticas culturales de sus países de origen hacen también aportes importantes a la nueva sociedad. (Portes 1995, Tilly 1990). El valor de las relaciones y redes sociales entre individuos de un mismo grupo étnico o nacional reside en la capacidad de sus miembros para desarrollar recursos potenciales en el nuevo país. Estos recursos, sostiene Mines (1981), tienden a mejorar y aumentar con el apoyo de nuevos grupos de inmigrantes. Portes y Sensenbrenner (1993) reconocen los efectos positivos de estas relaciones entre grupos de inmigrantes, especialmente en algunas transacciones económicas y en la reducción de contratos formales en actividades de trabajo.

Esto que bien podría considerarse un tipo de capital social sustentado en gran medida por una relación de mutua confianza e interacciones interpersonales, constituye muchas veces una función importante en el mantenimiento de una red social que facilita el acceso a un trabajo y en la diseminación de información sobre otras oportunidades en la nueva comunidad de muchos inmigrantes. Esto se logra, en gran medida, a la proximidad física y residencial de familias y grupos de inmigrantes en un mismo barrio o sector geográfico en una ciudad. Borjas y Tienda (1992), demuestran, por ejemplo, que muchas veces los inmigrantes están dispuestos a pagar un precio relativamente alto en relación a sus bajos ingresos a cambio de una mayor proximidad residencial con miembros de grupos étnicos similares a los que ellos pertenecen.

Otros autores indican haber encontrado en los EE.UU. resultados similares entre inmigrantes Hispanos pertenecientes a grupos con educación e ingresos más altos, entre los cuales se observó una cierta resistencia a cambiar de ciudad, vecindario, o 
área geográfica, donde posiblemente perderían sus relaciones de amistad y pertenencia a su comunidad étnica o nacional (Edwards et al. 1993: 132). Hay también otros investigadores quienes plantean una tesis de asimilación residencial diferente y sostienen que tanto entre los hispanos como entre los miembros de otros grupos, se puede observar un mayor grado de movilidad habitacional hacia otras áreas residenciales a medida que alcanzan niveles de ingreso y posición social más altos.

La concentración residencial de algunos grupos de inmigrantes refleja y responde a ciertas manifestaciones de segregación racial o étnica como lo indica Massey (1993). Este autor señala el hecho que la alta concentración de inmigrantes latinos en ciertas áreas de Estados Unidos, puede disminuir la necesidad de algunos inmigrantes para aprender inglés y acentuaría el aislamiento de estos individuos (Massey, 1995).

\section{Los conceptos de Capital Social y Capital Cultural}

Coleman (1988) define el concepto de capital social como un factor de la estructura social que permite a los individuos y a la sociedad alcanzar objetivos específicos. Entre los elementos constitutivos del capital social se destaca principalmente el grado de confianza con que los individuos se relacionan y actúan junto a otros, desarrollando así una conducta de solidaridad y reciprocidad con otros miembros del grupo social.

Según esta tesis, se podría argumentar que entre los miembros de un mismo grupo nacional o étnico sería posiblemente más factible encontrar los elementos básicos del "capital social" debido a un mayor sentido de pertenencia, solidaridad y experiencias culturales comunes. Investigadores como Marsden (1988) y Friedman y Krackhardt (1997) se refieren a una condición de homofilia social para denominar estos elementos comunes entre individuos de una misma etnia o nacionalidad.

El concepto de "capital cultural" fue inicialmente utilizado por Pierre Bourdieu y Jean "Claude Passeron (1977) para referirse a los signos y elementos utilizados por miembros de un grupo humano en el proceso de selección socio-cultural en sus actividades de vida. Bordieu (1984) utiliza este concepto en el análisis de movimientos demográficos y específicamente en el estudio de los factores de exclusión de las poblaciones minoritarias por parte de los grupos o sectores dominantes. Una de las conclusiones de Bordieu es que en todo sistema social los grupos dominantes hacen uso del capital cultural para es- tablecer la distancia o proximidad cultural, o para incorporar o excluir nuevos miembros. Según esta tesis, una parte importante del "capital cultural" en toda sociedad, está representado por las normas jurídicas, los patrones culturales, las fronteras residenciales, las barreras de organización y los servicios exclusivos para los grupos dominantes.

\section{La inmigración Made in USA}

A través de su historia, los Estados Unidos de Norteamérica ha sido un país predominante abierto y receptor de una numerosa inmigración internacional. Durante los últimos dos siglos, individuos de otros continentes y diversas naciones llegaron a radicarse en este país. Sólo en el período comprendido entre 1830 y 1930 se estima que más de 40 millones de inmigrantes llegaron a los Estados Unidos y, en la actualidad, un número superior a los 100 millones de norteamericanos, es decir, casi el $40 \%$ de la población total del país, son descendientes de esos inmigrantes. Esta proporción tiene la tendencia a seguir creciendo si se consideran los 700,000 inmigrantes que llegan cada año a los Estados Unidos.

El impacto de este proceso migratorio bien puede apreciarse en numerosas formas y expresiones culturales de la sociedad norteamericana. La composición demográfica de los diferentes estados varía según el número de sus habitantes que han nacido en otros países. Este es el caso de California donde más de un $25 \%$ de su población ha nacido en otro país, lo es también el caso de Nueva York con el 18\% y siguen Texas y Arizona, con una proporción superior al 10\% de sus habitantes nacidos en el extranjero, respectivamente.

Las estadísticas sobre migración revelan, sin embargo, un importante cambio. En las últimas décadas ha habido una progresiva reducción en el número de inmigrantes provenientes de Europa mientras el número de personas originarias de México, América Latina, Asia y África ha crecido significativamente. Hacia comienzos de esta década se observa que más de la mitad de los inmigrantes que entraban a los Estados Unidos eran originarios de sólo de 10 países. Entre estas naciones, un tercio de ellas eran de Asia y más de un cuarto (26\%) de México, Centro América y el Caribe. Junto con este cambio en la procedencia nacional de los inmigrantes se observa también un marcado incremento en la diversidad étnica de ellos pero sin un cambio significativo en el nivel de oportunidades o de aceptación de los nuevos inmigrantes. 
Entre algunos de los problemas de incorporación que tienen muchos de los nuevos inmigrantes al país del norte, se destacan algunas opiniones y expresiones de rechazo a formas culturales diferentes, cierto grado de segregación residencial en barrios y sectores urbanos, acceso limitado a una buena educación, trabajo y oportunidades para los inmigrantes y sus familias.

Muchas de las actitudes y demostraciones de rechazo hacia algunos inmigrantes han sido atribuidas a una falta de información y conocimiento de la cultura y aportes laborales y económicos de los inmigrantes a la nueva sociedad.

La creencia que los inmigrantes no trabajan o no contribuyen a la economía nacional es ampliamente rechazada por los hechos y las cifras de varias investigaciones. Por ejemplo, un informe del Consejo de Asesores Económicos de la Casa Blanca (2007) concluyó que la inmigración en su conjunto aumenta el Producto Nacional Bruto en aproximadamente US\$ 37 billones de dólares al año. Ese mismo año (2007), un estudio realizado por el Instituto de Políticas Públicas del estado de Nueva York concluyo que los inmigrantes en ese estado aportaban una productividad económica equivalente a US $\$ 229$ billones de dólares al estado de Nueva York. Dicha cifra corresponde al 22,4\% del Producto Estatal Bruto. A nivel nacional, ya a comienzos de esta década el economista Alan Greenspan, ex - Presidente de la Reserva Federal de EE.UU., informaba que los trabajadores inmigrantes, sin documentos, "donaban" al país la suma de US\$ 31 billones de dólares anuales, por concepto de impuestos Federales y Estatales que les eran descontados de sus salarios, pero sin tener ningún derecho a recibir algún beneficio o servicio a cambio. Por esta razón, la actual administración del gobierno y el Congreso de los Estados Unidos han planteado la necesidad de aprobar una reforma inmigratoria que facilite la legalización de trabajadores no documentados cuyo número se estima aproximadamente en el 5\% de la fuerza laboral del país.

\section{La presencia hispana en los Estados Unidos}

Los hispanos o latinos, (como se denomina en EE.UU. a personas de origen español o provenientes de América Latina), constituyen uno de los grupos étnicos de mayor crecimiento en ese país. Actualmente, su número total se aproxima a los 50 millones de personas y podrían alcanzar los 100 millones en el año 2050. Las proyecciones demográficas sugieren que en las próximas cuatro décadas, el 25\% de la población norteamericana, es decir, uno de cada cuatro habitantes podría ser de origen hispano.

A diferencia de otros grupos de inmigrantes, la presencia de los latinos en los Estados Unidos no es un fenómeno reciente. Muy por el contrario, si se considera que hace más de 150 años, los estados de California, Texas, Arizona, Nueva México, Utah y Colorado eran territorios mexicanos. Texas se independizó de México y se anexó a los Estados Unidos en 1845. Un año más tarde, en 1846, como resultado de la guerra entre Estados Unidos y México, el país del norte incorporó y anexó el resto de los estados mencionados. Además de estas relaciones históricas y territoriales, existe una alta proporción de latinos nacidos en los Estados Unidos y que toman parte activa en la vida del país. Este es el caso, por ejemplo, del $62 \%$ de hispanos de ascendencia mexicana y de numerosas personas que han venido de Puerto Rico, de Cuba y otros menos numerosos procedentes de América Central y América del Sur. En años recientes, la inmigración de hispanos a los Estados Unidos ha aumentado debido, principalmente, a un mayor número de inmigrantes provenientes de México, el Caribe y América Central. La distribución de los latinos en los Estados Unidos revela que casi el $87 \%$ de ellos se encuentra radicado en no más de diez estados. Entre ellos, los que tienen un porcentaje más alto de latinos son California (34\%), Texas (19\%), Nueva York (9\%), Florida (7\%), Illinois (4\%), y Arizona, New México y New Jersey con 3\% cada uno.

Más allá del impacto demográfico, la presencia de los hispanos en los Estados Unidos se puede apreciar también en otras áreas como son la economía, la política, las artes, educación, deportes y otras instituciones y expresiones culturales. El poder de compra de los hispanos, por ejemplo, se estima actualmente en casi $\$ 1$ trillón de dólares anuales, lo que en una economía de consumo constituye un poder y contribución importante. En el año 2008, se estimaba que una proporción superior al 50\% de los hispanos tenía habitualmente acceso y hacía uso de internet para información y comunicación. Se observa también que la mayoría de los hispanos (más del 60\%) son jóvenes menores de 34 años de edad lo cual explica, en parte, que sea el grupo étnico más numeroso y de más rápido crecimiento en los Estados Unidos.

Las nuevas generaciones de latinos comienzan a participar activamente en diversos campos profesionales, política y otras áreas. Por primera vez en la historia del país el nombramiento de Sonia Sotomayor como Jueza de la Corte Suprema, pone de relieve la "pre- 
sencia hispana" en una de las más altas instituciones del país. También en el mundo de la política se comienza a escuchar con más frecuencia el concepto denominado el factor latino, haciendo referencia a la importancia que los hispanos se registren en el nuevo Censo de población del 2010 y logren inscribir más de 3 millones de nuevos votantes.

A pesar del rápido crecimiento demográfico y los numerosos aportes de latinos en los Estados Unidos, es importante no ignorar los problemas y desafíos que afectan a numerosos miembros de esta comunidad hispana. En gran medida, se trata de problemas derivados de una estructura socioeconómica donde aún existe una alta desigualdad de ingresos y persistente pobreza. A diferencia de las políticas de apoyo a la migración europea del pasado, las condiciones actuales para los inmigrantes se caracterizan por una creciente limitación de oportunidades. A partir de la década de 1960, se observa que al variar la composición de nacionalidad y etnia de la inmigración a EE.UU. -cuando por vez primera el número de inmigrantes europeos es inferior a los provenientes de México, América Latina y Asia-, se origina también una disminución en programas y servicios tradicionalmente ofrecidas a inmigrantes europeos. Las áreas de mayor impacto y que requieren urgente atención son las relacionadas con educación, trabajo e ingresos.

\section{(a) Educación}

El sistema educativo norteamericano aunque ofrece grandes ventajas comparativas respecto a otros países, aún no ha logrado romper la barrera de las desigualdades tanto de acceso a la educación como en relación a la calidad de la enseñanza. Las diferencias entre los sectores público y privado de escuelas y distritos se mantienen tanto en lo referente a recursos como a resultados escolares.

Hace poco más de una década, un informe nacional elaborado por una Comisión para la Excelencia en la Educación de Hispano-Americanos (1998) señalaba la gravedad de los problemas educacionales de acceso y progreso académico que afectaba a numerosos latinos en los Estados Unidos. En años recientes, la situación no ha mejorado del todo y uno de los indicadores que aún persiste es la alta tasa de deserción escolar de un $28 \%$ que afecta a jóvenes hispanos entre 16 y 24 años de edad. Este nivel de deserción escolar es casi el doble que la tasa estimada para alumnos Afro-Americanos y tres veces más alta que entre los estudiantes blancos. Esta situación de bajo acceso y rendimiento educacional tiende lógicamente a repercutir en las bajas proporciones de jóvenes latinos que terminan exitosamente la educación secundaria, que luego ingresan a las universidades y logran una formación profesional y técnica competitiva en el mundo laboral.

\section{(b) Trabajo}

La relación entre educación y trabajo es claramente reconocida en la sociedad contemporánea.

Aquellos con más educación tienden a encontrar trabajos mejor remunerados y con mayor probabilidad de retención que quienes no han logrado un buen nivel educacional. Pese a esto, entre los grupos de familias hispanas en los EE.UU. se registra un alto nivel de participación laboral de varios de sus miembros.

Aproximadamente el 90\% o más de todos los trabajadores migrantes ocupados en la agricultura y agro-industria norteamericana son hispanos. Muchas veces, como lo revelan numerosos informes, tanto sus condiciones de vida y los servicios que reciben son deficientes.

Un elevado número de niños, hijos de los trabajadores migrantes, que oscila entre los 500.000 a 800.000 niños, participan también activamente en algunos trabajos agrícolas en las granjas que los emplean. Muchos de estos niños están sujetos a diferentes tipos de accidentes laborales y con peligro a enfermedades por el uso de pesticidas y otros productos químicos.

Asimismo, se ha observado que a causa del uso de aguas contaminadas, la incidencia de infecciones parasitarias y enfermedades gastrointestinales entre los trabajadores agrícolas migrantes y sus familias, se estima ser 35 veces más alta que los niveles registrados para el resto de la población. Muchos de estos trabajadores tienen alto riesgo de sufrir problemas dentales, nutrición deficiente y accidentes laborales. Sus hijos son también vulnerables a tener altos niveles de mortalidad y problemas de salud que pueden incidir en su desarrollo físico y social.

\section{(c) Ingresos}

La distribución de los ingresos en un país ha sido considerada como uno de los factores que afecta con mayor fuerza a los grupos económicamente más vulnerables y consolida la pobreza.

En los Estados Unidos los ingresos acusan un alto grado de desigualdad medido por el coeficiente de Gini estimado en .33, en una escala que oscila 
entre valores de 0 , para condiciones de igualdad total, a 1 para designar desigualdad absoluta.

Según este indicador, la desigualdad de los ingresos en EE.UU. es bastante más alta que la registrada en algunos países europeos como Holanda, Bélgica, Suiza, Suecia y Noruega donde el valor del coeficiente Gini es menor a 30 .

A fines del 2009 la tasa de pobreza en Estados Unidos alcanzaba al 13,2\% de la población total. Sin embargo, los grupos más afectados son los hispanos con una tasa del 23.2\% y los Afro-Americanos con un nivel de pobreza del 24,7\%.

La pobreza, independientemente de raza, color o nacionalidad, tiende a promover y estimular una serie de problemas sociales que afecta finalmente a todos los grupos y sectores. Del mismo modo, cualquier tipo de discriminación o prácticas de exclusión contra grupos minoritarios terminan por dañar al sistema social en tu totalidad.

La discriminación hacia algunos hispanos en los Estados Unidos ha sido documentada no sólo en las áreas relacionadas con el trabajo y los ingresos sino también en lo que respecta al acceso a préstamos, a la salud, y a otros servicios. En relación al acceso a servicios de salud, se estima que más de un tercio de los latinos en EEUU carecen de un seguro de salud que pueda cubrir sus necesidades básicas. Esta situación es aún más crítica entre los trabajadores agrícolas migrantes y sus familias. Estas personas, debido a la movilidad del empleo, la falta de conocimiento del sistema, la ausencia de servicios rurales, y la falta de dinero para pagar atención médica, son más vulnerables en caso de cualquiera enfermedad o accidente. La ironía de esta realidad es que pese a que Estados Unidos destina más del 12\% del Producto Domestico Neto a gastos en salud, todavía hay más de 40 millones de habitantes -incluyendo a muchos hispanos- que no disponen de un mínimo seguro médico.

\section{El proceso de migración internacional en Chile}

\section{La inmigración a Chile}

A lo largo de su historia Chile también se ha caracterizado como un país abierto a los procesos de migración internacional. Según datos del Censo Demográfico de 2002, la población total del país superaba los 15 millones de habitantes y los inmigrantes extranjeros residentes en Chile era de 184.464 personas. Es decir, la proporción de inmigrantes en relación a la población total es relativamente baja y alcanza sólo al 1,2 \%. (Instituto Nacional de Estadísticas, Chile).

El número de inmigrantes en Chile y su incidencia porcentual en la población total del país ha tenido algunas variaciones importantes en el curso de los últimos 50 años. Muchos de estos cambios obedecen principalmente a las características socio-demográficas de los migrantes, las condiciones laborales, sociales y políticas tanto del país de origen como la situación en Chile.

Martínez (2005) en su estudio sobre la "magnitud y dinámica de la inmigración en Chile" informa que, según el Censo de Población de 1952, Chile tenía una población de casi 6 millones de habitantes y un número de inmigrantes de 103.878 personas. De acuerdo a estos datos, la proporción de inmigrantes en el país alcanzaba al $1.75 \%$ de la población total. Treinta años más tarde, en 1982, cuando la población del país superaba los 11 millones, los inmigrantes eran 84.345 personas, lo que equivalía a una proporción del $0.75 \%$ de la población total. Esta proporción, como se ha indicado anteriormente, sube en el año 2002 al 1.22\% de la población total del país. Este crecimiento porcentual es relativamente modesto si se considera que aún es inferior a la proporción de inmigrantes de $1.75 \%$ de la población nacional en1952.

De acuerdo a estas cifras, la incidencia porcentual de la inmigración en Chile es baja especialmente si se la compara con otros países donde el porcentaje de inmigrantes en relación a la población total es mayor. Tal es el caso de Argentina donde los inmigrantes representan el 4\% del total; Costa Rica el 8\%; Estados Unidos el 12\%, y Canadá el 18\% de todos los habitantes de ese país.

Norambuena (2004) observa que el crecimiento económico junto a la estabilidad democrática y política del país durante los últimos 20 años, han contribuido al aumento del número de inmigrantes especialmente aquellos provenientes de países vecinos como Perú, Bolivia y Argentina, donde han tenido durante este lapso de tiempo crisis económicas y políticas de consideración.

Los residentes extranjeros en Chile provienen mayoritariamente de América (77,1\%), seguidos por inmigrantes de Europa (17,2 \%); Asia (4,2\%); África $(0,7 \%)$ y de Oceanía $(0,8 \%)$. Datos censales indican que el mayor número de los inmigrantes en Chile proceden de países de América del Sur $(67,9 \%)$ entre los cuales se incluyen las siguientes nacionalidades: argentinos, 48.176; peruanos, 37.860; bolivianos, 10.919; ecuatorianos, 9.393; 
brasileños, 6.895; venezolanos, 4.338; colombianos, 4.095; uruguayos, 2.241; paraguayos, 1.222; y 22 de otros países no especificados. Inmigrantes provenientes de América del Norte incluye a 7.753 estadounidenses, 1.845 canadienses y 1.697 mexicanos. Entre los inmigrantes de origen europeo se destacan 9.084 españoles; 5.473 alemanes; 3.927 italianos y 3.043 franceses.

La diversidad de origen o nacionalidad de los inmigrantes radicados en Chile ha constituido un aporte cultural y económico importante para el país. De manera similar, se podría argumentar que lo mismo ocurre en los lugares de destino a donde han emigrado cerca de un millón de chilenos.

\section{La emigración de Chile}

De acuerdo a información de Naciones Unidas los emigrantes internacionales de Chile han establecido su nueva residencia en diversos países de cada continente. Del total de emigrantes chilenos el 50\% de ellos residen en países de América Latina y el Caribe; el 20,6\% en países de América del Norte incluyendo México, Canadá y Estados Unidos, y otro 20\% viven en Europa.

Estos datos revelan también que casi nueve de cada diez chilenos que salieron de Chile y que hoy día residen en el exterior, se encuentran preferentemente en países con un nivel de desarrollo humano alto $(49,5 \%)$ y muy alto (45,3\%). (PNUD, 2009)

En la mayoría de los casos, estos chilenos han contado con un alto grado de aceptación por parte de los pobladores de sus nuevos lugares de residencia y han podido surgir en sus respectivas ocupaciones y comunidades. Por esta razón, despierta curiosidad y cierto malestar observar expresiones negativas hacia algunos grupos de inmigrantes en Chile.

\section{Estereotipos y exclusión}

Por ejemplo, un caso reciente ocurrido en el país, llamó la atención cuando un dirigente político y candidato a diputado por Santiago, afirmara que era necesario expulsar de Chile a los ciudadanos peruanos que no tuviesen la documentación exigida. Entre los argumentos dados por este personero para justificar la deportación de estos nuevos inmigrantes, se encuentran precisamente algunos de los estereotipos mencionados anteriormente por el informe de Naciones Unidas. Menciona, por ejemplo,--sin presentar datos o pruebas válidas--, que "la gran cantidad de residentes ilegales peruanos en la comuna (de Santiago) ha provocado que muchas personas (chilenos) no puedan recibir la atención médica necesaria en el servicio de salud público." Esto, a su juicio, provoca "un grave problema de convivencia" entre los chilenos y los extranjeros...

Se debe recordar, sin embargo, que el número total de peruanos residentes en Chile, con documentación en orden, alcanza -según el último Censo de Población- a menos de 38.000 personas en un país donde hay actualmente más de 16 millones de habitantes. Según esto, se puede estimar con alta probabilidad que el número de peruanos sin documentación, o con documentación aún en trámite, ha de ser una mínima fracción del total y su impacto a nivel nacional es claramente insignificante. Aparentemente, lo que ocurriría en este caso, es más bien un problema de alta visibilidad de los inmigrantes peruanos como lo demuestra Stefoni (2004), en un análisis sobre la formación de comunidades peruanas en Santiago. Según este estudio, durante la década de 1992 y 2002 la inmigración peruana habría tenido un explosivo crecimiento del 394 \% además de una alta visibilidad en el sector de Plaza de Armas, en centro de la ciudad de Santiago. Las cifras indican que del total de inmigrantes peruanos en Chile (37.860), no menos de 27,739 de ellos, es decir una proporción no menor al $73 \%$ viven en algunas de las comunas de Santiago, lo que contribuye ciertamente a su notoria presencia en esta ciudad capital. Del mismo modo, vale recordar que los inmigrantes peruanos queriendo legalizar su permanencia en Chile, han sido los que más rápido se acogieron al beneficio de una visa de residencia temporaria concedido por el gobierno de la Presidente Michelle Bachelet a quienes no disponían de documentación migratoria al día. En esta oportunidad, como lo indican Riquelme, J. y Alarcón, G. (2008), un total de 32 mil peruanos recibieron sus respectivas visas.

Por esta razón, la respuesta del Gobierno de Chile a la proposición de deportar a peruanos sin documentos no se dejo esperar. El ministro secretario general de la Presidencia de la República, José Antonio Viera-Gallo declaró rápidamente que la propuesta era "tan lamentable como si en Argentina se dijera que hubiera que deportar a los 200 mil chilenos que viven allá". A continuación agregó:

"Vivimos en un mundo en que los habitantes migran. Por mucho tiempo, décadas, los chilenos migraron a Argentina y Argentina los acogió, les dio trabajo, les dio oportunidades para vivir. Ahora tenemos el caso inverso, en que hay personas que migran hacia nuestro país y no veo por qué Chile no va a tener la misma actitud que en su minuto 
tuvo Argentina. Nosotros -concluyó- tenemos que acostumbrarnos a una política migratoria, en que respetamos los derechos de los migrantes".

\section{Conclusiones}

Como bien lo señala el Informe sobre el Desarrollo Humano 2009 y lo ratifican las palabras del ministro chileno, las percepciones erróneas no aportan nada positivo a la discusión del tema de la inmigración internacional. Los estereotipos negativos que muestran a los inmigrantes como personas que 'nos quitan el empleo' y 'viven a costa de nuestros impuestos', -aunque abundan en los medios de comunicación y en la opinión pública, especialmente en épocas de crisis económica,- no tienen realmente validez ni una base empírica seria en donde sustentarse.

El informe del Programa de Naciones Unidas para el Desarrollo invita a un diálogo amplio sobre este tema y propone algunos objetivos que aseguren la relación entre migración y el desarrollo humano. Entre estos propósitos y actividades directamente relacionadas con los procesos migratorios internacionales se destacan las siguientes:

1. Velar por el respeto y garantías de los derechos humanos de los migrantes;

2. Facilitar y apoyar la existencia de oportunidades de una vida digna para los migrantes;

3. Reducir los costos económicos y humanos involucrados en la migración;

4. Elaborar procedimientos que favorezcan a los migrantes y a las comunidades de la nueva residencia de los inmigrantes y sus familias;

5. Desarrollar políticas públicas que permitan fortalecer las relaciones entre los procesos de migración y las necesidades, modelos y estrategias de desarrollo.

Estos objetivos representan por cierto un fuerte desafío para las ciencias sociales y el Trabajo Social en la búsqueda de nuevas perspectivas conceptuales y teóricas sobre la inmigración. Esto implica poder establecer un vínculo más estrecho entre la investigación y la difusión de informaciones, que ayuden a eliminar falsas concepciones sobre los inmigrantes. Asimismo, es necesario diseñar una clara relación entre los procesos migratorios y las políticas públicas para el desarrollo social.

Como bien lo indica Serani (2001), el flujo migratorio que recibió Chile durante las últimas décadas "encontró a un país que institucionalmente no estaba preparado para recibirlos, carente de políticas migratorias y de programas de inserción, lo que significó que en 1998 existieran 40 mil extranjeros en condición de irregularidad, los cuales se atuvieron a un proceso de regularización migratoria."

Esta situación, sin embargo, no es sólo exclusiva para el caso de Chile sino que se encuentra también presente en los Estados Unidos y en Europa. En esos países, como en el nuestro, se requiere de renovados esfuerzos para atender y solucionar los problemas derivados de la inmigración internacional.Son temas y desafíos ciertamente globales que, para lograr resultados efectivos, necesitan de modelos, políticas y soluciones que consideren e involucren a todos los miembros y componentes del sistema social.

\section{Referencias}

ALBA, R. AND CHAMLIN. M.B. (1983) "Ethnic Identification among Whites", American Sociological Review, 48:240-47.

APONTE, R. (2000) "Latinos and Political Interventions". Working paper for Latinos 2000. Interdisciplinary Approaches to the Millennium (February 3-6, 2000), Indiana University, Purdue University at Indianapolis.

BERRY, J.W. (1993) "Ethnic Identity in Plural Societies." In: M. E. Bernal and G. P. Knight (Eds.). Ethnic Identity. Formation and Transmission Among Hispanics and Other Minorities. PP. 271-296. New York: State University of New York Press.

BORJAS, G. AND TIENDA. M. (1987) "The Economic Consequences of Immigration." Science 235: 645651.

BOURDIEU, PIERRE. (1984). Distinction: A Social Critique of the Judgment of Taste. Cambridge: Harvard University Press.

BOURDIEU, PIERRE AND PASSERON. J.C. (1977). Reproduction in Education, Society, and Culture. London: Sage Productions.

CASTELlS, M. (1975) "Immigrant Workers and Class Struggle in Advanced Capitalism: The Western European Experiences."

COLEMAN, J. S. (1988). "Social Capital in the Creation of Human Capital." The American Journal of Sociology 94: S95-S120.

EDWARDS, J., ROSEFELD, P., THOMAS, P., AND THOMAS, M. (1993). "Willingness to Relocate for Employment: A Survey of Hispanics, Non-Hispanic Whites, and Blacks." Hispanic Journal of Behavioral Sciences 15: 121-33.

FRIEDMAN, R. AND KRACKHARDT. D. (1997) "Social Capital and Career Mobility." Journal of Applied Behavioral Science 33:316-34. 
GLAZER, N. AND MOYNIHAN. D. (1970) Beyond the Melting Pot: The Negroes, Puerto Ricans, Jews, Italians and Irish in New York City. Cambridge, Mass.: M. I. T. Press.

GORDON, M. (1964) Assimilation in American Life: The Role of Race, Religion, and National Origins. New York: Oxford University Press.

HANDLIN, O. (1941) Boston's Immigrants: A Study of Acculturation, Cambridge: Harvard University Press.

JARET, C. (1999) Troubled by Newcomers: Anti-Immigrant Attitudes and Action during Two Eras of Mass Immigration to the United States. Journal of American Ethnic History. 18 (3): 9-37.

MARTíNEZ, PJ. (2005). Magnitud y dinámica de la inmigración en Chile según el censo de 2002. Papeles de Población, Abril-Junio, número 044. Universidad Autónoma del Estado de México, Tolula, México. (pp.109-147).

MASSEY, D. (1993) "Latinos, Poverty, and the Underclass: A New Agenda for Research." Hispanic Journal of Behavioral Sciences 15 (4), 449-475.

MASSEY, D. (1995) "The New Immigration and Ethnicity in the United States." Population and Development Review 21: 631-52.

MINES, R. (1981) Developing a Community Tradition of Migration. A Field Study in Rural Zacatecas: Mexico and California Settlement Areas. Monograph Series, No. 3 La Jolla, Calif. Center for US-Mexico Studies. San Diego: University of California.

MYRDAL, G. (1944) An American Dilemma; The Negro Problem and Modern Democracy. New York: Harper. New York Newsday. October, 1991.

NORAMBUENA, C. (2004) "Chile y sus Nuevos Inmigrantes. Ni acogidos ni rechazados". Revista Universitaria, \#85 (8 de Mayo), Universidad de Santiago de Chile, Santiago, Chile.

PARRA-LUNA, F. (2000) "A Model Measuring the Performance of Social Systems" (pp.89-118). In The Performance of Social Systems, edited by F. Parra-Luna. New York: Kluwer Academic/Plenum Publishers.

PORTES, A. (ed.) (1995) The Economic Sociology of Immigration: Essays on Networks, Ethnicity, and Entrepreneurship. New York: Russel Sage Foundation.

PORTES, A. (1978) "Migration and Underdevelopment." Politics and Society 8: 1-48.

PORTES, A. AND SENSENBRENNER. J. (1993) "Embeddedness and Immigration: Notes on the Social Determinants of Economic Action." American Journal of Sociology 98, 6: 1320-50.

PORTES, A. AND MANNING. R.D. (1986) "The Immigrant Enclave: Theory and Empirical Examples." In S. Olzak and J. Nagel (Eds.), Competitive Ethnic Relations, Academic Press, Inc., Orlando.
RIQUELME, R.J. Y ALARCÓN, M.G. (2008) "El peso de la historia en la inmigración peruana en Chile", Polis: Revista académica de la Universidad Bolivariana, Santiago, Chile.

SASSEN-KOOB, S. (1978) "The International Circulation of Resources and Development: The Case of Migrant Labor." Development and Change 9 : 509-545.

SERANI, P.E. (2001) Políticas Migratorias, Biblioteca del Congreso Nacional, Dpto. de Estudios, Extensión y Publicaciones. DEPESEX/BCN/SERIE ESTUDIOS, AÑO XI, N²57, Santiago, Chile.

STEFONI, S. (2004) "Inmigración y Ciudadanía: La Formación de Comunidades Peruanas en Santiago y la Emergencia de nuevos ciudadanos". Política \#43, pp. 319-336

TILLY, C. (1990) "Transplanted Networks." In: Virginia Yans-McLaughlin (Ed.), Immigration Reconsidered: History, Sociology, and Politics. PP. 79-95. New York: Oxford University Press.

PROGRAMA DE NACIONES UNIDAS PARA EL DESARROLLO (PNUD), Informe sobre el Desarrollo Humano 2009. Cuadro B: Emigrantes internacionales por lugar de residencia (pg. 161).

INSTITUTO NACIONAL DE ESTADÍSTICA, (INEChile). Censo de Población, 2002. 